\title{
Existence of positive solutions for a generalized and fractional ordered Thomas-Fermi theory of neutral atoms
}

Wenquan Feng, Shurong Sun ${ }^{*}$ and Ying Sun

"Correspondence:

sshrong@163.com

School of Mathematical Science,

University of Jinan, Jinan, Shandong

250022, P.R. China

\section{Abstract}

The singular boundary value problem we discuss is as follows:

$$
\begin{array}{ll}
{ }^{C} D_{0^{+}}^{\alpha} u(t)=\lambda q(t) f(t, u(t)), & 0<t<1, \\
\alpha_{1} u(0)+\alpha_{2} u^{\prime}(0)=a, & \beta_{1} u(1)+\beta_{2} u^{\prime}(1)=b,
\end{array}
$$

where $1<\alpha \leq 2, \lambda\rangle 0$ is a parameter, ${ }^{C} D_{0^{+}}^{\alpha}$ is the Caputo fractional derivative. We present the existence of positive solutions for a fractional boundary value problem modeled from the Thomas-Fermi equation subjected to Sturm-Liouville boundary conditions.

MSC: $34 \mathrm{~A} 08 ; 34 \mathrm{~B} 16 ; 34 \mathrm{~B} 18$

Keywords: fractional differential equation; singular boundary value problem; positive solution; Thomas-Fermi theory

\section{Introduction}

The Thomas-Fermi model (see [1-3]), named after Llewellyn Thomas and Enrico Fermi, is a quantum mechanical theory of electronic structure within many-body systems. In 1927, Thomas and Fermi independently conceived this statistical model applying it to approximate the distribution of electrons in an atom. The Thomas-Fermi model leads to the nonlinear second order differential equation

$$
t^{\frac{1}{2}} y^{\prime \prime}=y^{\frac{3}{2}}
$$

The boundary conditions under discussion include three circumstances as follows:

(i) for the neutral atom with Bohr radius $\rho$, the boundary conditions are

$$
y(0)=1, \quad \rho y^{\prime}(\rho)=y(\rho)
$$

(ii) for the ionized atom, the boundary conditions are

$$
y(0)=1, \quad y(\rho)=0
$$

(c) 2015 Feng et al. This article is distributed under the terms of the Creative Commons Attribution 4.0 International License (http://creativecommons.org/licenses/by/4.0/), which permits unrestricted use, distribution, and reproduction in any medium, provided you give appropriate credit to the original author(s) and the source, provide a link to the Creative Commons license, and indicate if changes were made. 
(iii) for the isolated neutral atom, the boundary conditions are

$$
y(0)=1, \quad \lim _{x \rightarrow \infty} y(x)=0
$$

As we know fractional calculus has played a more and more significant role in engineering, science technology, economy and other fields (see [4-13]) just owing to the reason that fractional derivatives could not only have the equation much briefer but also lead to a better description that seems closer to reality. Standing on this point, our theory applies to the generalized equation

$$
\begin{aligned}
& { }^{\mathrm{C}} D_{0^{+}}^{\alpha} u(t)=\lambda q(t) f(t, u(t)), \quad 0<t<1, \\
& \alpha_{1} u(0)+\alpha_{2} u^{\prime}(0)=a, \quad \beta_{1} u(1)+\beta_{2} u^{\prime}(1)=b,
\end{aligned}
$$

where we suppose

(I) $1<\alpha \leq 2, \alpha_{i}, \beta_{i} \geq 0, i=1,2, \lambda>0, \frac{\beta_{1}}{\beta_{1}+\beta_{2}}<\frac{\alpha_{1}}{\alpha_{2}} \leq 1, f:[0,1] \times[0,+\infty) \rightarrow(0,+\infty)$ is a given continuous function, $q:(0,1] \rightarrow(0,+\infty)$ is continuous and $0<\int_{0}^{1} q(t) d t<+\infty$.

In order to get a better understanding of our work, let us retrospect some recent attractive results on the existence of positive solutions for boundary value problems.

Agarwal and O'Regan [14] discussed the two-point boundary value problem

$$
\begin{array}{ll}
y^{\prime \prime}=q f(t, y), & 0<t<a, \\
y(0)=a_{0}, & k y^{\prime}(a)=y(a), \quad k \geq a,
\end{array}
$$

where $a>0$ is fixed. They presented an upper and lower solution theory for boundary value problems modeled from the Thomas-Fermi equation subjected to boundary conditions related to the neutral atom with Bohr radius.

Zhang [15] considered the existence and multiplicity of positive solutions for the nonlinear fractional differential equation boundary value problem

$$
\begin{array}{ll}
D_{0^{+}}^{\alpha} u(t)=f(t, u(t)), & 0<t<1, \\
u(0)+u^{\prime}(0)=0, & u(1)+u^{\prime}(1)=0,
\end{array}
$$

where $1<\alpha \leq 2$ is a real number, $D_{0^{+}}^{\alpha}$ is the Caputo fractional derivative, $f:[0,1] \times$ $(0,+\infty) \rightarrow(0,+\infty)$ is a given continuous function. Their results are based on a fixed point theorem on cones.

Sun and Wen [16] investigated the nonlinear third order ordinary equation

$$
u^{\prime \prime \prime}(t)=\lambda a(t) f(u(t)), \quad 0<t<1,
$$

with the boundary conditions

$$
\alpha u^{\prime}(0)+\beta u^{\prime \prime}(0)=0, \quad u(1)=u^{\prime}(1)=0 .
$$

Via using the properties of Green's function and Guo-Krasnosel'skii's fixed point theorem, Zhao et al. [17] studied the existence of positive solutions for the following boundary 
value problem:

$$
\begin{array}{ll}
D_{0^{+}}^{\alpha} u(t)=\lambda f(u(t)), & 0<t<1, \\
u(0)+u^{\prime}(0)=0, & u(1)+u^{\prime}(1)=0 .
\end{array}
$$

Su and Zhang [18] proved an existence result for the problem

$$
\begin{aligned}
& { }^{\mathrm{C}} D_{0^{+}}^{\alpha} u(t)=f\left(t, u(t),{ }^{\mathrm{C}} D_{0^{+}}^{\beta} u(t)\right), \quad 0<t<1, \\
& a_{1} u(0)-a_{2} u^{\prime}(0)=A, \quad b_{1} u(1)+b_{2} u^{\prime}(1)=B,
\end{aligned}
$$

where $1<\alpha \leq 2,0<\beta \leq 1, a_{i}, b_{i} \geq 0, i=1,2, a_{1} b_{1}+a_{1} b_{2}+a_{2} b_{1}>0,{ }^{\mathrm{C}} D_{0^{+}}^{\alpha}$ is the Caputo fractional derivative and $f:[0,1] \times(0,+\infty) \rightarrow(0,+\infty)$ is a given continuous function.

Zhai and $\mathrm{Xu}$ [19] established the existence and uniqueness of positive solutions to a class of four-point boundary value problem of Caputo fractional differential equations for any given parameter:

$$
\begin{aligned}
& { }^{\mathrm{C}} D_{0^{+}}^{\alpha} u(t)+\lambda f(t, u(t))=0, \quad 0<t<1, \\
& u^{\prime}(0)-\mu_{1} u(\xi)=0, \quad u^{\prime}(1)+\mu_{2} u(\eta)=0,
\end{aligned}
$$

where $1<\alpha \leq 2,0 \leq \xi \leq \eta \leq 1,0 \leq \mu_{1}, \mu_{2} \leq 1, \lambda>0$ is a parameter, $f(t, x):[0,1] \times R^{+} \rightarrow$ $R^{+}$is continuous and increasing in $x$ for each $t \in[0,1]$.

Moreover, there are also many interesting research works on the existence of solutions of boundary value problems, readers can refer to [20-25].

After reading the previous results, it hits on the head that we might turn to problem (1.1). Now let us have a look at the novel contributions of this work. First, we generalize the Thomas-Fermi equation, the second order ordinary differential equation, into a fractional order. Second, $f$ is a function varying along with two variables $t$ and $u$ instead of being constrained by only one variable $u$. Third, the problem is a singular one, as $q(t)$ may be singular at $t=0$. Last but not the least, the existence and multiplicity of the positive solutions depend on a parameter $\lambda$.

This paper is organized as follows. In Section 2, we retrospect some preliminaries and lemmas. In Section 3, our results about the existence of positive solutions of problem (1.1) are obtained. The multiplicity of existence of positive solutions for problem (1.1) is debated in Section 4.

\section{Preliminaries}

For the convenience of the readers, we give some background material from fractional calculus theory to facilitate analysis of boundary value problem (1.1).

Definition 2.1 ([4]) The Riemann-Liouville fractional integral of order $\alpha>0$ of a function $y:(0,+\infty) \rightarrow \mathbb{R}$ is given by

$$
I_{0^{+}}^{\alpha} y(t)=\frac{1}{\Gamma(\alpha)} \int_{0}^{t}(t-s)^{\alpha-1} y(s) d s
$$

provided the right-hand side is pointwise defined on $(0,+\infty)$. 
Definition 2.2 ([4]) The Caputo fractional derivative of order $\alpha>0$ of a continuous function $y:(0,+\infty) \rightarrow \mathbb{R}$ is given by

$$
{ }^{\mathrm{C}} D_{0^{+}}^{\alpha} y(t)=\frac{1}{\Gamma(n-\alpha)} \int_{0}^{t} \frac{y^{(n)}(s)}{(t-s)^{\alpha-n+1}} d s
$$

where $n$ is the smallest integer greater than or equal to $\alpha$, provided the right-hand side is pointwise defined on $(0,+\infty)$.

Lemma 2.1 ([5]) The Caputo derivative of a power function is

$$
{ }^{\mathrm{C}} D_{0^{+}}^{\alpha} t^{\mu}=\mu(\mu-1) \cdots(\mu-n+1) \frac{\Gamma(1+\mu-n)}{\Gamma(1+\mu-\alpha)} t^{\mu-\alpha} \quad \text { for } t \in(0, \infty),
$$

given, in particular, ${ }^{C} D_{0^{+}}^{\alpha} t^{\mu}=0, \mu=0,1, \ldots, n-1$, where ${ }^{\mathrm{C}} D_{0^{+}}^{\alpha}$ is the Caputo fractional derivative, $n$ is the smallest integer greater than or equal to $\alpha$.

There are some reduced properties between the Riemann-Liouville type fractional derivative and the Caputo one.

Lemma 2.2 ([6]) If $\alpha=n$ is an integer, the Caputo fractional derivative of order $\alpha$ is the usual derivative of order $n$. Additionally, the following properties are well known: $I_{0^{+}}^{\alpha} I_{0^{+}}^{\beta} f(t)=I_{0^{+}}^{\alpha+\beta} f(t),{ }^{\mathrm{C}} D_{0^{+}}^{\alpha} I_{0^{+}}^{\alpha} f(t)=f(t), \alpha>0, f \in C[0,1]$.

From the definition of Caputo derivative and Lemma 2.1, we can obtain the following statement.

Lemma 2.3 ([4]) Let $\alpha>0$. Then the fractional differential equation

$$
{ }^{\mathrm{C}} D_{0^{+}}^{\alpha} u(t)=0
$$

has a unique solution

$$
u(t)=c_{0}+c_{1} t+c_{2} t^{2}+\cdots+c_{n-1} t^{n-1}, \quad c_{i} \in \mathbb{R}, i=0,1,2, \ldots, n-1,
$$

where $n$ is the smallest integer greater than or equal to $\alpha$.

Lemma 2.4 ([4]) Let $\alpha>0$. Assume that $u \in C^{n}[0,1]$. Then

$$
I_{0^{+}}^{\alpha}{ }^{C} D_{0^{+}}^{\alpha} u(t)=u(t)+c_{0}+c_{1} t+c_{2} t^{2}+\cdots+c_{n-1} t^{n-1}
$$

for some $c_{i} \in \mathbb{R}, i=0,1,2, \ldots, n-1$, where $n$ is the smallest integer greater than or equal to $\alpha$.

With the help of Lemmas 2.1, 2.3 and 2.4, we present the Green's function for boundary value problem of fractional differential equation. 
Lemma 2.5 Let $h \in C(0,1) \cap L(0,1)$ and $1<\alpha \leq 2$. Take $\Delta=\alpha_{2} \beta_{1}-\alpha_{1} \beta_{1}-\alpha_{1} \beta_{2}<0$. Then the fractional differential equation boundary value problem

$$
\begin{aligned}
& { }^{\mathrm{C}} D_{0^{+}}^{\alpha} u(t)=h(t), \quad 0<t<1, \\
& \alpha_{1} u(0)+\alpha_{2} u^{\prime}(0)=0, \quad \beta_{1} u(1)+\beta_{2} u^{\prime}(1)=0
\end{aligned}
$$

has a unique solution

$$
u(t)=\int_{0}^{1} G(t, s) h(s) d s
$$

where

$$
G(t, s)= \begin{cases}\frac{(t-s)^{\alpha-1}}{\Gamma(\alpha)}+\left(\alpha_{1} t-\alpha_{2}\right)\left(\frac{\beta_{1}(1-s)^{\alpha-1}}{\Delta \Gamma(\alpha)}+\frac{\beta_{2}(1-s)^{\alpha-2}}{\Delta \Gamma(\alpha-1)}\right), & 0<s \leq t<1, \\ \left(\alpha_{1} t-\alpha_{2}\right)\left(\frac{\beta_{1}(1-s)^{\alpha-1}}{\Delta \Gamma(\alpha)}+\frac{\beta_{2}(1-s)^{\alpha-2}}{\Delta \Gamma(\alpha-1)}\right), & 0<t \leq s<1 .\end{cases}
$$

Proof We may apply Lemma 2.4 to reduce equation (2.1) to an equivalent integral formula

$$
\begin{aligned}
u(t) & =I_{0^{+}}^{\alpha} h(t)-c_{0}-c_{1} t \\
& =\frac{1}{\Gamma(\alpha)} \int_{0}^{t}(t-s)^{\alpha-1} h(s) d s-c_{0}-c_{1} t, \quad c_{0}, c_{1} \in \mathbb{R} .
\end{aligned}
$$

Then

$$
u^{\prime}(t)=\frac{1}{\Gamma(\alpha-1)} \int_{0}^{t}(t-s)^{\alpha-2} h(s) d s-c_{1} .
$$

From $\alpha_{1} u(0)+\alpha_{2} u^{\prime}(0)=0, \beta_{1} u(1)+\beta_{2} u^{\prime}(1)=0$, we have

$$
\begin{aligned}
& c_{0}=\frac{\alpha_{2} \beta_{1}}{\Delta \Gamma(\alpha)} \int_{0}^{1}(1-s)^{\alpha-1} h(s) d s+\frac{\alpha_{2} \beta_{2}}{\Delta \Gamma(\alpha-1)} \int_{0}^{1}(1-s)^{\alpha-2} h(s) d s, \\
& c_{1}=-\frac{\alpha_{1} \beta_{1}}{\Delta \Gamma(\alpha)} \int_{0}^{1}(1-s)^{\alpha-1} h(s) d s-\frac{\alpha_{1} \beta_{2}}{\Delta \Gamma(\alpha-1)} \int_{0}^{1}(1-s)^{\alpha-2} h(s) d s .
\end{aligned}
$$

Therefore, the unique solution of problem (2.1) is

$$
\begin{aligned}
u(t)= & \frac{1}{\Gamma(\alpha)} \int_{0}^{t}(t-s)^{\alpha-1} h(s) d s+\frac{\alpha_{1} \beta_{1} t-\alpha_{2} \beta_{1}}{\Delta \Gamma(\alpha)} \int_{0}^{1}(1-s)^{\alpha-1} h(s) d s \\
& +\frac{\alpha_{1} \beta_{2} t-\alpha_{2} \beta_{2}}{\Delta \Gamma(\alpha-1)} \int_{0}^{1}(1-s)^{\alpha-2} h(s) d s .
\end{aligned}
$$

The proof is complete.

Lemma 2.6 Let $1<\alpha \leq 2$. Then the following fractional differential equation boundary value problem

$$
\begin{aligned}
& { }^{\mathrm{C}} D_{0^{+}}^{\alpha} u(t)=0, \quad 0<t<1, \\
& \alpha_{1} u(0)+\alpha_{2} u^{\prime}(0)=a, \quad \beta_{1} u(1)+\beta_{2} u^{\prime}(1)=b
\end{aligned}
$$


has a unique solution

$$
u(t)=\frac{\left(\beta_{1}+\beta_{2}\right) a-\alpha_{2} b+\left(\alpha_{1} b-\beta_{1} a\right) t}{-\Delta} .
$$

In the following discussion, we denote

$$
\varphi(t):=\frac{\left(\beta_{1}+\beta_{2}\right) a-\alpha_{2} b+\left(\alpha_{1} b-\beta_{1} a\right) t}{-\Delta} .
$$

Next we present some useful properties of the Green's function.

Lemma 2.7 The Green's function $G(t, s)$ obtained in Lemma 2.5 has these properties as follows:

(i) $G(t, s) \in C([0,1] \times[0,1))$ and $G(t, s)>0$ for $t, s \in(0,1)$;

(ii) there exists a positive function $\gamma \in C(0,1)$ such that

$$
\min _{t \in\left[\frac{1}{4}, \frac{3}{4}\right]} G(t, s) \geq \gamma(s) M(s), \quad \max _{t \in[0,1]} G(t, s) \leq M(s), \quad s \in(0,1)
$$

where

$$
M(s)=-\frac{\left(\alpha_{1} \beta_{1}+\alpha_{1} \beta_{2}\right)(1-s)^{\alpha-1}}{\Delta \Gamma(\alpha)}-\frac{\alpha_{2} \beta_{2}(1-s)^{\alpha-2}}{\Delta \Gamma(\alpha-1)}, \quad \gamma(s) \geq \frac{1}{4} .
$$

Proof From the expression of $G(t, s)$, it is clear that $G(t, s) \in C([0,1] \times[0,1))$ and $G(t, s)>0$ for $s, t \in(0,1)$.

Here we define

$$
\begin{aligned}
& g_{1}(t, s)=\frac{(t-s)^{\alpha-1}}{\Gamma(\alpha)}+\left(\alpha_{1} t-\alpha_{2}\right)\left(\frac{\beta_{1}(1-s)^{\alpha-1}}{\Delta \Gamma(\alpha)}+\frac{\beta_{2}(1-s)^{\alpha-2}}{\Delta \Gamma(\alpha-1)}\right), \quad s \leq t, \\
& g_{2}(t, s)=\left(\alpha_{1} t-\alpha_{2}\right)\left(\frac{\beta_{1}(1-s)^{\alpha-1}}{\Delta \Gamma(\alpha)}+\frac{\beta_{2}(1-s)^{\alpha-2}}{\Delta \Gamma(\alpha-1)}\right), \quad t \leq s .
\end{aligned}
$$

Then, obviously, $g_{1}(t, s)$ is a continuous function for $t \in\left[\frac{1}{4}, \frac{3}{4}\right]$ and $g_{2}(t, s)$ is decreasing with respect to $t$. Hence, we have

$$
\begin{aligned}
& g_{1}(t, s) \geq g_{2}(t, s) \geq \min _{t \in\left[\frac{1}{4}, \frac{3}{4}\right]} g_{2}(t, s) \\
&=\left(\frac{3}{4} \alpha_{1}-\alpha_{2}\right)\left(\frac{\beta_{1}(1-s)^{\alpha-1}}{\Delta \Gamma(\alpha)}+\frac{\beta_{2}(1-s)^{\alpha-2}}{\Delta \Gamma(\alpha-1)}\right) \text { for } t \in\left[\frac{1}{4}, \frac{3}{4}\right] \\
& \max _{t \in[0,1]} g_{1}(t, s) \leq \frac{\left(\beta_{1}\left(\alpha_{1} t-\alpha_{2}\right)+\Delta\right)(1-s)^{\alpha-1}}{\Delta \Gamma(\alpha)}+\frac{\left(\alpha_{1} t-\alpha_{2}\right) \beta_{2}(1-s)^{\alpha-2}}{\Delta \Gamma(\alpha-1)} \\
& \leq \frac{\left(-\beta_{1} \alpha_{2}+\Delta\right)(1-s)^{\alpha-1}}{\Delta \Gamma(\alpha)}+\frac{-\alpha_{2} \beta_{2}(1-s)^{\alpha-2}}{\Delta \Gamma(\alpha-1)} \\
&=\frac{\left(-\alpha_{1} \beta_{1}-\alpha_{1} \beta_{2}\right)(1-s)^{\alpha-1}}{\Delta \Gamma(\alpha)}+\frac{-\alpha_{2} \beta_{2}(1-s)^{\alpha-2}}{\Delta \Gamma(\alpha-1)} \\
& \max _{t \in[0,1]} g_{2}(t, s)=g_{2}(0, s)=-\frac{\alpha_{2} \beta_{1}(1-s)^{\alpha-1}}{\Delta \Gamma(\alpha)}-\frac{\alpha_{2} \beta_{2}(1-s)^{\alpha-2}}{\Delta \Gamma(\alpha-1)} \\
& \leq \frac{\left(-\alpha_{1} \beta_{1}-\alpha_{1} \beta_{2}\right)(1-s)^{\alpha-1}}{\Delta \Gamma(\alpha)}+\frac{-\alpha_{2} \beta_{2}(1-s)^{\alpha-2}}{\Delta \Gamma(\alpha-1)}
\end{aligned}
$$


Thus, we get

$$
\begin{aligned}
& \min _{t \in\left[\frac{1}{4}, \frac{3}{4}\right]} G(t, s) \geq m(s):=\left(\frac{3}{4} \alpha_{1}-\alpha_{2}\right)\left(\frac{\beta_{1}(1-s)^{\alpha-1}}{\Delta \Gamma(\alpha)}+\frac{\beta_{2}(1-s)^{\alpha-2}}{\Delta \Gamma(\alpha-1)}\right), \quad s \in[0,1), \\
& \max _{t \in[0,1]} G(t, s) \leq M(s):=-\frac{\left(\alpha_{1} \beta_{1}+\alpha_{1} \beta_{2}\right)(1-s)^{\alpha-1}}{\Delta \Gamma(\alpha)}-\frac{\alpha_{2} \beta_{2}(1-s)^{\alpha-2}}{\Delta \Gamma(\alpha-1)}, \quad s \in[0,1) .
\end{aligned}
$$

Let

$$
\gamma(s)=\frac{m(s)}{M(s)}=\frac{\left(\frac{3}{4} \alpha_{1}-\alpha_{2}\right)\left(\frac{\beta_{1}(1-s)^{\alpha-1}}{\Delta \Gamma(\alpha)}+\frac{\beta_{2}(1-s)^{\alpha-2}}{\Delta \Gamma(\alpha-1)}\right)}{-\frac{\left(\alpha_{1} \beta_{1}+\alpha_{1} \beta_{2}\right)(1-s)^{\alpha-1}}{\Delta \Gamma(\alpha)}-\frac{\alpha_{2} \beta_{2}(1-s)^{\alpha-2}}{\Delta \Gamma(\alpha-1)}} .
$$

From hypothesis (I), we see

$$
\begin{aligned}
\gamma(s) & \geq \frac{\left(\frac{3}{4} \alpha_{1}-\alpha_{2}\right)\left(\frac{\beta_{1}(1-s)^{\alpha-1}}{\Delta \Gamma(\alpha)}+\frac{\beta_{2}(1-s)^{\alpha-2}}{\Delta \Gamma(\alpha-1)}\right)}{-\frac{\alpha_{2} \beta_{1}(1-s)^{\alpha-1}}{\Delta \Gamma(\alpha)}-\frac{\alpha_{2} \beta_{2}(1-s)^{\alpha-2}}{\Delta \Gamma(\alpha-1)}} \\
& =\frac{\alpha_{2}-\frac{3}{4} \alpha_{1}}{\alpha_{2}} \geq \frac{1}{4} .
\end{aligned}
$$

Hence the statements in Lemma 2.7 are proved.

Our main results are typically based on the following fixed point theorem.

Lemma 2.8 ([7]) Let $B$ be a Banach space, and let $K \subset B$ be a cone. Assume that $\Omega_{1}, \Omega_{2}$ are open and bounded subset of $B$ with $0 \in \Omega_{1}, \bar{\Omega}_{1} \subset \Omega_{2}$, and let $T: K \cap\left(\bar{\Omega}_{2} \backslash \Omega_{1}\right) \rightarrow K$ be a completely continuous operator such that

(i) $\|T u\| \leq\|u\|, u \in K \cap \partial \Omega_{1}$ and $\|T u\| \geq\|u\|, u \in K \cap \partial \Omega_{2}$ or

(ii) $\|T u\| \geq\|u\|, u \in K \cap \partial \Omega_{1}$ and $\|T u\| \leq\|u\|, u \in K \cap \partial \Omega_{2}$.

Then $T$ has a fixed point in $K \cap\left(\bar{\Omega}_{2} \backslash \Omega_{1}\right)$.

\section{Existence results}

In this section, we establish the existence of positive solutions for problem (1.1) in terms of different values of the parameter $\lambda$.

From Lemmas 2.5 and 2.6, we know that proving the existence of positive solutions for fractional differential boundary value problem (1.1) is equivalent to discussing the existence of positive solutions for the problem

$$
\begin{aligned}
& { }^{\mathrm{C}} D_{0^{+}}^{\alpha} \nu(t)=\lambda q(t) f(t, v(t)+\varphi(t)), \quad 0<t<1, \\
& \alpha_{1} v(0)+\alpha_{2} v^{\prime}(0)=0, \quad \beta_{1} v(1)+\beta_{2} v^{\prime}(1)=0,
\end{aligned}
$$

then $u=v+\varphi$ must be the solution of problem (1.1), and vice versa.

Let $X=C^{1}[0,1]$ with norm $\|u\|=\max _{t \in[0,1]}|u(t)|, u \in X$. Clearly, $X$ is a Banach space. Take

$$
P=\left\{u \in X \mid u(t) \geq 0, \min _{t \in\left[\frac{1}{4}, \frac{3}{4}\right]} u(t) \geq \frac{1}{4}\|u\|\right\} .
$$

Then it is easy to see that $P$ is a positive cone in $X$. 
Define an operator $T: P \rightarrow X$ by

$$
T v(t)=\lambda \int_{0}^{1} G(t, s) q(s) f(s, v(s)+\varphi(s)) d s, \quad 0 \leq t \leq 1, v \in P .
$$

Noting that the possible singular point of $q$ is 0 and $M$ maybe singular only at 1 , we are able to show that $\int_{0}^{1} M(s) q(s) d s$ is well defined by hypothesis (I), Lemma 2.7 and the convergence of $\int_{0}^{1} M(s) d s$ and $\int_{0}^{1} q(s) d s$. From this, it is easy to see that the operator $T$ is also well defined.

Denote

$$
I_{1}=\frac{1}{16} \int_{\frac{1}{4}}^{\frac{3}{4}} M(s) q(s) d s, \quad I_{2}=\int_{0}^{1} M(s) q(s) d s .
$$

Lemma 3.1 Assume that (I) holds. Then the pair of equations (3.1) and (3.2) is equivalent to the nonlinear integral equation

$$
v(t)=\lambda \int_{0}^{1} G(t, s) q(s) f(s, v(s)+\varphi(s)) d s
$$

In other words, every solution of (3.1) and (3.2) is also a continuous solution of (3.3) and vice versa.

Proof Let $v \in X$ be a solution of (3.1) and (3.2); as the same method of proving Lemma 2.5, we can get that $v$ is a solution of (3.3).

On the other hand, let $v$ be a continuous solution of $(3.3)$ on $[0,1]$. Then define

$$
\begin{aligned}
\omega(t)= & \lambda \int_{0}^{1} G(t, s) q(s) f(s, v(s)+\varphi(s)) d s \\
= & \lambda\left(\frac{1}{\Gamma(\alpha)} \int_{0}^{t}(t-s)^{\alpha-1} q(s) f(s, v(s)+\varphi(s)) d s\right. \\
& +\frac{\alpha_{1} \beta_{1} t-\alpha_{2} \beta_{1}}{\Delta \Gamma(\alpha)} \int_{0}^{1}(1-s)^{\alpha-1} q(s) f(s, v(s)+\varphi(s)) d s \\
& \left.+\frac{\alpha_{1} \beta_{2} t-\alpha_{2} \beta_{2}}{\Delta \Gamma(\alpha-1)} \int_{0}^{1}(1-s)^{\alpha-2} q(s) f(s, v(s)+\varphi(s)) d s\right) \\
= & \lambda\left(I_{0^{+}}^{\alpha} q(t) f(t, v(t)+\varphi(t))+\frac{\alpha_{1} \beta_{1} t-\alpha_{2} \beta_{1}}{\Delta} I_{0^{+}}^{\alpha} q(1) f(1, v(1)+\varphi(1))\right. \\
& \left.+\frac{\alpha_{1} \beta_{2} t-\alpha_{2} \beta_{2}}{\Delta} I_{0^{+}}^{\alpha-1} q(1) f(1, v(1)+\varphi(1))\right) .
\end{aligned}
$$

From Lemmas 2.1 and 2.2, we have

$$
\begin{aligned}
\omega^{\prime}(t)= & \lambda\left(D_{0^{+}}^{1} I_{0^{+}}^{1} I_{0^{+}}^{\alpha-1} q(t) f(t, v(t)+\varphi(t))+\frac{\alpha_{1} \beta_{1}}{\Delta} I_{0^{+}}^{\alpha} q(1) f(1, v(1)+\varphi(1))\right. \\
& \left.+\frac{\alpha_{1} \beta_{2}}{\Delta} I_{0^{+}}^{\alpha-1} q(1) f(1, v(1)+\varphi(1))\right) \\
= & \lambda\left(I_{0^{+}}^{\alpha-1} q(t) f(t, v(t)+\varphi(t))+\frac{\alpha_{1} \beta_{1}}{\Delta} I_{0^{+}}^{\alpha} q(1) f(1, v(1)+\varphi(1))\right.
\end{aligned}
$$




$$
\begin{aligned}
+ & \left.\frac{\alpha_{1} \beta_{2}}{\Delta} I_{0^{+}}^{\alpha-1} q(1) f(1, v(1)+\varphi(1))\right), \\
{ }^{C} D_{0^{+}}^{\alpha} \omega(t) & =D_{0^{+}}^{\alpha}\left(\omega(t)-\omega\left(0^{+}\right)-\omega^{\prime}\left(0^{+}\right)\right)=D_{0^{+}}^{\alpha} I_{0^{+}}^{\alpha} \lambda q(t) f(t, v(t)+\varphi(t)) \\
& =\lambda q(t) f(t, v(t)+\varphi(t)) .
\end{aligned}
$$

One can verify easily that $\alpha_{1} v(0)+\alpha_{2} v^{\prime}(0)=0, \beta_{1} v(1)+\beta_{2} v^{\prime}(1)=0$. Therefore, $v$ is a continuous solution of (3.1) and (3.2). The proof is completed.

Lemma 3.1 indicates that the solution of problems (3.1) and (3.2) coincides with the fixed point of the operator $T$.

Incidentally, we have the following dispensable lemma at hand.

Lemma 3.2 The operator $T: P \rightarrow P$ is completely continuous.

Proof Obviously, $T v(t) \geq 0$. Then first we are going to prove that the operator $T: P \rightarrow X$ is continuous. Actually, for each $v_{n}, v \in P$ and $v_{n} \rightarrow v$, we get

$$
\begin{aligned}
\left\|T v_{n}-T v\right\| & =\max _{t \in[0,1]}\left|\lambda \int_{0}^{1} G(t, s) q(s)\left(f\left(s, v_{n}(s)+\varphi(s)\right)-f(s, v(s)+\varphi(s))\right) d s\right| \\
& \leq \lambda \max _{t \in[0,1]} \int_{0}^{1}|G(t, s) q(s)| f\left(s, v_{n}(s)+\varphi(s)\right)-f(s, v(s)+\varphi(s)) \mid d s \\
& =\lambda \int_{0}^{1} \max _{t \in[0,1]} G(t, s) q(s)\left|f\left(s, v_{n}(s)+\varphi(s)\right)-f(s, v(s)+\varphi(s))\right| d s .
\end{aligned}
$$

Since $f$ is continuous, for any $\varepsilon>0$, there exists $N \in \mathbb{N}$ such that $f\left(s, v_{n}(s)+\varphi(s)\right)-f(s, v(s)+$ $\varphi(s)) \mid<\varepsilon$, when $n>N$. Hence,

$$
\begin{aligned}
\left\|T v_{n}-T \nu\right\| & \leq \varepsilon \lambda \int_{0}^{1} \max _{t \in[0,1]} G(t, s) q(s) d s \\
& \leq \varepsilon \lambda \int_{0}^{1} M(s) q(s) d s=\varepsilon \lambda I_{2} \quad \text { for all } n>N
\end{aligned}
$$

So it is sufficient to say that $T$ is continuous.

For $v \in P$, by Lemma 2.7 , we have

$$
\begin{aligned}
\min _{t \in\left[\frac{1}{4}, \frac{3}{4}\right]} T v(t) & =\min _{t \in\left[\frac{1}{4}, \frac{3}{4}\right]} \lambda \int_{0}^{1} G(t, s) q(s) f(s, v(s)+\varphi(s)) d s \\
& \geq \lambda \int_{0}^{1} \min _{t \in\left[\frac{1}{4}, \frac{3}{4}\right]} G(t, s) q(s) f(s, v(s)+\varphi(s)) d s \\
& \geq \frac{1}{4} \lambda \int_{0}^{1} M(s) q(s) f(s, v(s)+\varphi(s)) d s .
\end{aligned}
$$

On the other hand,

$$
\|T v\|=\max _{t \in[0,1]}|T v(t)| \leq \int_{0}^{1} \lambda M(s) q(s) f(s, v(s)+\varphi(s)) d s .
$$


Thus, we obtain

$$
\min _{t \in\left[\frac{1}{4}, \frac{3}{4}\right]} T v(t) \geq \frac{1}{4}\|T v\|
$$

therefore, $T: P \rightarrow P$.

Let $\Omega \subset P$ be bounded. Then there exists a positive constant $Q>0$ such that $\|v\| \leq$ $Q$ for all $v \in \Omega$. Take $L=\max _{t \in[0,1],\|v\| \leq Q}|f(t, v(t)+\varphi(t))|+1$. Then, for each $v \in \Omega$, from Lemma 2.7, we have

$$
\begin{aligned}
|T v(t)| & \leq \int_{0}^{1}|\lambda G(t, s) q(s) f(s, v(s)+\varphi(s))| d s \\
& \leq \lambda L \int_{0}^{1} M(s) q(s) d s=\lambda L I_{2} .
\end{aligned}
$$

Hence, $T(\Omega)$ is bounded.

Next we are about to prove that for all $\varepsilon>0$, each $v \in P, t_{1}, t_{2} \in[0,1], t_{1}<t_{2}$, let $\eta=$ $\min \left\{\frac{1}{2}, \frac{\varepsilon}{L_{1}}\right\}$, we have $\left|T v\left(t_{2}\right)-T v\left(t_{1}\right)\right|<\varepsilon$, when $t_{2}-t_{1}<\eta$. In fact, for each $v \in \Omega$, we have

$$
\begin{aligned}
(T v)^{\prime}(t)= & \lambda\left(\frac{1}{\Gamma(\alpha-1)} \int_{0}^{t}(t-s)^{\alpha-2} q(s) f(s, v(s)+\varphi(s)) d s\right. \\
& +\frac{\alpha_{1} \beta_{1}}{\Delta \Gamma(\alpha)} \int_{0}^{1}(1-s)^{\alpha-1} q(s) f(s, v(s)+\varphi(s)) d s \\
& \left.+\frac{\alpha_{1} \beta_{2}}{\Delta \Gamma(\alpha-1)} \int_{0}^{1}(1-s)^{\alpha-2} q(s) f(s, v(s)+\varphi(s)) d s\right) \\
\leq & \left(\frac{\lambda L}{\Gamma(\alpha-1)}+\frac{\lambda \alpha_{1} \beta_{2} L}{\Delta \Gamma(\alpha-1)}\right) \int_{0}^{1}(1-s)^{\alpha-2} q(s) d s+\frac{\lambda \alpha_{1} \beta_{1} L}{\Delta \Gamma(\alpha)} \int_{0}^{1} q(s) d s .
\end{aligned}
$$

Since we have proved $\int_{0}^{1}(1-s)^{\alpha-2} q(s) d s<\infty$ and $\int_{0}^{1} q(s) d s<\infty$, we can just define

$$
L_{1}=\left(\frac{\lambda L}{\Gamma(\alpha-1)}+\frac{\lambda \alpha_{1} \beta_{2} L}{\Delta \Gamma(\alpha-1)}\right) \int_{0}^{1}(1-s)^{\alpha-2} q(s) d s+\frac{\lambda \alpha_{1} \beta_{1} L}{\Delta \Gamma(\alpha)} \int_{0}^{1} q(s) d s
$$

Then $(T v)^{\prime}(t) \leq L_{1}$.

Whence,

$$
\left|T \nu\left(t_{2}\right)-T v\left(t_{1}\right)\right| \leq L_{1}\left(t_{2}-t_{1}\right)<\varepsilon .
$$

By the Arzela-Ascoli theorem, the operator $T: P \rightarrow P$ is completely continuous. The proof is completed.

For convenience, we give some denotations:

$$
\begin{aligned}
& F_{0}=\limsup _{v \rightarrow 0^{+}} \max _{t \in[0,1]} \frac{f(t, v+\varphi(t))}{v}, \quad F_{\infty}=\limsup _{v \rightarrow+\infty} \max _{t \in[0,1]} \frac{f(t, v+\varphi(t))}{v}, \\
& f_{0}=\liminf _{v \rightarrow 0^{+}} \min _{t \in[0,1]} \frac{f(t, v+\varphi(t))}{v}, \quad f_{\infty}=\liminf _{v \rightarrow+\infty} \min _{t \in[0,1]} \frac{f(t, v+\varphi(t))}{v} .
\end{aligned}
$$


Theorem 3.1 Assume that $\left(I_{1} f_{\infty}\right)^{-1}=0$ if $f_{\infty}=\infty$ and $\left(I_{2} F_{0}\right)^{-1}=\infty$ if $F_{0}=0$. If $I_{1} f_{\infty}>I_{2} F_{0}$ holds, then for each

$$
\lambda \in\left(\left(I_{1} f_{\infty}\right)^{-1},\left(I_{2} F_{0}\right)^{-1}\right),
$$

the boundary value problem (1.1) has at least one positive solution.

Proof Choose $\varepsilon>0$ sufficiently small such that

$$
\left(I_{1}\left(f_{\infty}-\varepsilon\right)\right)^{-1} \leq \lambda \leq\left(I_{2}\left(F_{0}+\varepsilon\right)\right)^{-1} .
$$

By the definition of $F_{0}$, we see that there exists $r_{1}>0$ such that

$$
f(t, v+\varphi(t)) \leq\left(F_{0}+\varepsilon\right) v \quad \text { for } 0<v \leq r_{1} .
$$

So if $v \in \partial P$ with $\|v\|=r_{1}$, then by (3.7) and (3.8), we see

$$
\begin{aligned}
\|T v\| & =\max _{t \in[0,1]}|T v(t)| \leq \int_{0}^{1} \lambda M(s) q(s) f(s, v(s)+\varphi(s)) d s \\
& \leq \int_{0}^{1} \lambda M(s) q(s)\left(F_{0}+\varepsilon\right) r_{1} d s \\
& \leq \lambda I_{2}\left(F_{0}+\varepsilon\right) r_{1} \leq r_{1}=\|v\| .
\end{aligned}
$$

Hence, if we choose $\Omega_{1}=\left\{v \in X:\|v\|<r_{1}\right\}$, then $\|T v\| \leq\|v\|$ for $v \in P \cap \partial \Omega_{1}$.

Let $r_{2}>0$ be a constant such that

$$
f(t, v+\varphi(t)) \geq\left(f_{\infty}-\varepsilon\right) v \quad \text { for } v \geq r_{2} .
$$

If $v \in \partial P$ with $\|v\|=r_{3}=\max \left\{2 r_{1}, r_{2}\right\}$, then by (3.7) and (3.9) we have

$$
\begin{aligned}
\|T v\| & =\max _{t \in[0,1]}|T v(t)| \geq \int_{\frac{1}{4}}^{\frac{3}{4}} \lambda G(t, s) q(s) f(s, v(s)+\varphi(s)) d s \\
& \geq \frac{1}{4} \lambda \int_{\frac{1}{4}}^{\frac{3}{4}} M(s) q(s)\left(f_{\infty}-\varepsilon\right) v(s) d s \\
& \geq \frac{1}{16} \lambda \int_{\frac{1}{4}}^{\frac{3}{4}} M(s) q(s)\left(f_{\infty}-\varepsilon\right)\|v\| d s \geq\|v\| .
\end{aligned}
$$

Thus, if we define $\Omega_{2}=\left\{v \in X:\|v\|<r_{3}\right\}$, then $\|T v\| \geq\|v\|$ for $v \in P \cap \partial \Omega_{2}$.

Then, by Lemma 2.8, we find that $T$ has a fixed point $v \in P \cap\left(\bar{\Omega}_{2} \backslash \Omega_{1}\right)$ with $r_{1} \leq\|v\| \leq r_{3}$, therefore, $v$ is a positive solution of (3.1) and (3.2), then $v+\varphi$ is a positive solution of (1.1). The proof is completed.

Theorem 3.2 Assume that $\left(I_{1} f_{0}\right)^{-1}=0$ iff $f_{0}=+\infty$ and $\left(I_{2} F_{\infty}\right)^{-1}=\infty$ if $F_{\infty}=0$. If $I_{1} f_{0}>I_{2} F_{\infty}$ holds, then for each

$$
\lambda \in\left(\left(I_{1} f_{0}\right)^{-1},\left(I_{2} F_{\infty}\right)^{-1}\right),
$$

the boundary value problem (1.1) has at least one positive solution. 
Proof Let $\lambda$ be endowed with (3.10) and $\varepsilon>0$ be such that

$$
\left(I_{1}\left(f_{0}-\varepsilon\right)\right)^{-1} \leq \lambda \leq\left(I_{2}\left(F_{\infty}+\varepsilon\right)\right)^{-1} .
$$

By the definition of $f_{0}$, we see that there exists $r_{1}>0$ such that

$$
f(t, v+\varphi(t)) \geq\left(f_{0}-\varepsilon\right) v \quad \text { for } 0<v \leq r_{1} .
$$

Hence, if $v \in \partial P$ with $\|v\|=r_{1}$, then by the same method used in Theorem 3.1, we get $\|T v\| \geq\|v\|$. Furthermore, if we choose $\Omega_{1}=\left\{v \in X:\|v\|<r_{1}\right\}$, then $\|T v\| \geq\|v\|$ for $v \in$ $P \cap \partial \Omega_{1}$. In the next, we choose $r_{2}>0$ subjected to

$$
f(t, v+\varphi(t)) \leq\left(F_{\infty}+\varepsilon\right) v \quad \text { for } v \geq r_{2} .
$$

We discuss two cases as follows.

Case 1. Assume that $f$ is bounded. Then there exists a certain constant $L>0$ such that $f(t, v+\varphi(t)) \leq L r_{3}$ for $v \in(0,+\infty)$. We define $r_{3}=\max \left\{2 r_{1}, r_{2}\right\}$ and $v \in \partial P$ with $\|v\|=r_{3}$, then

$$
\begin{aligned}
\|T u\| & =\max _{t \in[0,1]}|T v(t)| \leq \int_{0}^{1} \lambda M(s) q(s) f(s, v(s)+\varphi(s)) d s \\
& \leq \lambda L r_{3} \int_{0}^{1} M(s) q(s) d s \\
& \leq \lambda r_{3} I_{2} L \leq r_{3}=\|v\| .
\end{aligned}
$$

Therefore, $\|T v\| \leq\|v\|$ for $v \in \partial P_{r_{3}}=\left\{v \in P:\|v\|<r_{3}\right\}$.

Case 2. Assume that $f$ is unbounded. Then there exists some constant $r_{4}>\max \left\{2 r_{1}, r_{2}\right\}$ such that $f(t, v+\varphi(t)) \leq f\left(t, r_{4}+\varphi(t)\right)$ for $0<v \leq r_{4}$. Let $v \in \partial P$ with $\|v\|=r_{4}$. Then, from (3.11) and (3.13), we have

$$
\begin{aligned}
\|T v\| & =\max _{t \in[0,1]}|T v(t)| \leq \int_{0}^{1} \lambda M(s) q(s) f(s, v(s)+\varphi(s)) d s \\
& \leq \int_{0}^{1} \lambda M(s) q(s)\left(F_{\infty}+\varepsilon\right) r_{4} d s \\
& \leq \lambda I_{2}\left(F_{\infty}+\varepsilon\right) r_{4} \leq r_{4}=\|v\| .
\end{aligned}
$$

It is sufficient to show that $\|T v\| \leq\|v\|$ for $v \in \partial P_{r_{3}}=\left\{v \in P:\|v\|<r_{4}\right\}$. Then we take $\Omega_{2}=\left\{v \in X:\|v\|<r_{5}=: \max \left\{r_{3}, r_{4}\right\}\right\}$, then $\|T v\| \leq\|v\|$ for $v \in P \cap \partial \Omega_{2}$. From Lemma 2.8, operator $T$ has a fixed point $v \in P \cap\left(\bar{\Omega}_{2} \backslash \Omega_{1}\right)$ with $r_{1} \leq\|v\| \leq r_{5}$. It is clear enough that $v$ is a positive solution of (3.1) and (3.2), then $v+\varphi$ is a positive solution of (1.1). The proof is completed.

Theorem 3.3 Suppose that there exist constants $r_{2}>r_{1}>0$ for all $t \in[0,1]$, the following condition holds:

$$
\min _{\frac{1}{4} r_{1} \leq v \leq r_{1}} f(t, v+\varphi(t)) \geq \frac{r_{1}}{\lambda I_{1}}, \quad \max _{0 \leq \nu \leq r_{2}} f(t, v+\varphi(t)) \leq \frac{r_{2}}{\lambda I_{2}} .
$$

Then the boundary value problem (1.1) has at least one positive solution. 
Proof Choose $\Omega_{1}=\left\{v \in X:\|v\|<r_{1}\right\}$, then for $v \in P \cap \partial \Omega_{1}$, we have

$$
\begin{aligned}
\|T v\| & =\max _{t \in[0,1]}|T v(t)| \geq \min _{t \in\left[\frac{1}{4}, \frac{3}{4}\right]} \int_{\frac{1}{4}}^{\frac{3}{4}} \lambda G(t, s) q(s) f(s, v(s)+\varphi(s)) d s \\
& =\lambda \int_{\frac{1}{4}}^{\frac{3}{4}} \min _{t \in\left[\frac{1}{4}, \frac{3}{4}\right]} G(t, s) q(s) f(s, v(s)+\varphi(s)) d s \\
& \geq \frac{1}{4} \lambda \int_{\frac{1}{4}}^{\frac{3}{4}} M(s) q(s) f(s, v(s)+\varphi(s)) d s \\
& \geq \frac{1}{16} \lambda \int_{\frac{1}{4}}^{\frac{3}{4}} M(s) q(s) \min _{\frac{1}{4} r_{1} \leq v \leq r_{1}} f(s, v(s)+\varphi(s)) d s \\
& \geq \lambda I_{1} \frac{r_{1}}{\lambda I_{1}}=r_{1}=\|v\| .
\end{aligned}
$$

On the other hand, choose $\Omega_{2}=\left\{v \in X:\|v\|<r_{2}\right\}$, then for $v \in P \cap \partial \Omega_{2}$, we have

$$
\begin{aligned}
\|T v\| & =\max _{t \in[0,1]}|T v(t)| \leq \int_{0}^{1} \lambda M(s) q(s) f(s, v(s)+\varphi(s)) d s \\
& \leq \int_{0}^{1} \lambda M(s) q(s) \max _{0 \leq v \leq r_{2}} f(t, v(s)+\varphi(s)) d s \\
& \leq \lambda I_{2} \frac{r_{2}}{\lambda I_{2}}=r_{2}=\|v\| .
\end{aligned}
$$

Therefore from Lemma 2.8, operator $T$ has a fixed point $v \in P \cap\left(\bar{\Omega}_{2} \backslash \Omega_{1}\right)$ with $r_{1} \leq\|v\| \leq r_{2}$. It is clear enough that $v$ is a positive solution of (3.1) and (3.2), then $v+\varphi$ is a positive solution of (1.1). The proof is completed.

Theorem 3.4 Suppose that the following condition holds:

$$
\lambda I_{2} f(t, v+\varphi(t))<v \quad \text { for all } t \in[0,1], v \in(0,+\infty) .
$$

Then the boundary value problem (1.1) has no positive solution.

Proof Assume to the contrary that $v+\varphi$ is a positive solution of (1.1), $v$ is a positive solution of (3.1) and (3.2). Then

$$
\begin{aligned}
\|v\| & =\|T \nu\|=\max _{t \in[0,1]}|T v(t)| \leq \int_{0}^{1} \lambda M(s) q(s) f(s, v(s)+\varphi(s)) d s \\
& <\frac{1}{\lambda I_{2}}\|v\| \int_{0}^{1} \lambda M(s) q(s) d s=\|v\|,
\end{aligned}
$$

which comes to a contradiction and completes the proof.

Theorem 3.5 Suppose that the following condition holds:

$$
\lambda I_{1} f(t, v+\varphi(t))>v \quad \text { for all } t \in[0,1], v \in(0,+\infty) .
$$

Then the boundary value problem (1.1) has no positive solution. 
Proof The proof of Theorem 3.5 is similar to that of Theorem 3.4, therefore omitted.

Example 3.1 Consider the boundary value problem

$$
\begin{aligned}
& { }^{\mathrm{C}} D_{0^{+}}^{\frac{3}{2}} u(t)=\lambda t^{-\frac{1}{2}} u(t)(t+2+u(t)), \quad 0<t<1, \\
& u(0)=1, \quad u(1)=0 .
\end{aligned}
$$

Then

$$
F_{0}=2, \quad f_{\infty}=\infty, \quad F_{\infty}=\infty, \quad f_{0}=1 .
$$

By a direct calculation, we get

$$
I_{1} \approx 0.0369261219, \quad I_{2} \approx 1.7724538358
$$

From Theorem 3.1, we see if

$$
0=\frac{1}{I_{1} f_{\infty}}<\lambda<\frac{1}{I_{2} F_{0}} \approx 0.2820947942
$$

then problem (1.1) has at least one positive solution. From Theorem 3.4, if

$$
\lambda<\frac{1}{I_{2} F_{\infty}}=0,
$$

we see that problem (1.1) has no positive solution. By Theorem 3.5, if

$$
\lambda>\frac{1}{I_{1} f_{0}} \approx 27.0811000058
$$

then we obtain that problem (1.1) has no positive solution.

\section{Existence of multiple positive solutions}

Here we are about to give some results on the existence of multiple positive solutions. The following two lemmas will be of use to prove our main results.

Lemma 4.1 Assume that there exists a constant $c>0$ such that $\max _{0 \leq v \leq c} f(t, v+\varphi(t)) \leq \frac{c}{\lambda I_{2}}$ for all $t \in[0,1]$. Then

$$
\|T v\| \leq c \quad \text { for } v \in P \text { with }\|v\|=c .
$$

Proof If $v \in P$ with $\|v\|=c$, then

$$
\begin{aligned}
\|T v\| & =\max _{t \in[0,1]}|T v(t)| \leq \int_{0}^{1} \lambda M(s) q(s) f(s, v(s)+\varphi(s)) d s \\
& \leq \frac{c}{\lambda I_{2}} \int_{0}^{1} \lambda M(s) q(s) d s=c,
\end{aligned}
$$

the proof is complete. 
Lemma 4.2 Assume that there exists a constant $c>0$ such that $\min _{\frac{1}{4} c \leq v \leq c} f(t, v+\varphi(t)) \geq$ $\frac{c}{\lambda I_{1}}$ for all $t \in[0,1]$. Then

$$
\|T v\| \geq c \quad \text { for } v \in P \text { with }\|v\|=c .
$$

Proof If $v \in P$ with $\|v\|=c$, then for $t \in\left[\frac{1}{4}, \frac{3}{4}\right]$ we have $v(t) \geq \frac{1}{4}\|v\|$. Therefore, we have

$$
\begin{aligned}
\|T v\| & =\max _{t \in[0,1]}|T v(t)| \geq \min _{t \in\left[\frac{1}{4}, \frac{3}{4}\right]} \int_{\frac{1}{4}}^{\frac{3}{4}} \lambda G(t, s) q(s) f(s, v(s)+\varphi(s)) d s \\
& \geq \frac{1}{4} \lambda \int_{\frac{1}{4}}^{\frac{3}{4}} M(s) q(s) f(s, v(s)+\varphi(s)) d s \\
& \geq \frac{1}{16} \lambda \int_{\frac{1}{4}}^{\frac{3}{4}} M(s) q(s) \min _{\frac{1}{4} c \leq v \leq c} f(s, v(s)+\varphi(s)) d s \\
& \geq \lambda I_{1} \frac{c}{\lambda I_{1}}=c=\|v\|,
\end{aligned}
$$

so the proof is complete.

Theorem 4.1 Assume that there are constants $0<c_{1}<c_{2}<c_{3}<c_{4}$ such that

(i) $\max _{0 \leq \nu \leq c_{i}} f(t, v+\varphi(t)) \leq \frac{c_{i}}{\lambda I_{2}}$ for all $t \in[0,1], i=1,4$ and

(ii) $\min _{\frac{1}{4} c_{i} \leq v \leq c_{i}} f(t, v+\varphi(t)) \geq \frac{c_{i}}{\lambda I_{1}}$ for all $t \in[0,1], i=2,3$.

Then the boundary value problem (1.1) has at least two positive solutions.

Proof If

$$
\Omega_{i}=\left\{v \in X:\|v\|<c_{i}\right\}, \quad i=1,2,3,4
$$

then from Lemmas 4.1 and 4.2, we have

$$
\|T v\| \leq\|v\| \quad \text { for } v \in P \cap \partial \Omega_{i}, i=1,4
$$

and

$$
\|T v\| \geq\|v\| \quad \text { for } v \in P \cap \partial \Omega_{i}, i=2,3 .
$$

Now from Lemma 2.8 we get that $T$ has two fixed points, one in each of two sets $P \cap\left(\bar{\Omega}_{4} \backslash\right.$ $\left.\Omega_{3}\right)$ and $P \cap\left(\bar{\Omega}_{2} \backslash \Omega_{1}\right)$. This completes the proof.

In the same way, we can obtain the following result.

Theorem 4.2 Assume that there are constants $0<c_{1}<c_{2}<c_{3}<c_{4}$ such that

(i) $\max _{0 \leq v \leq c_{i}} f(t, v+\varphi(t)) \leq \frac{c_{i}}{\lambda I_{2}}$ for all $t \in[0,1], i=2,3$, and

(ii) $\min _{\frac{1}{4} c_{i} \leq v \leq c_{i}} f(t, v+\varphi(t)) \geq \frac{c_{i}}{\lambda I_{1}}$ for all $t \in[0,1], i=1,4$.

Then the boundary value problem (1.1) has at least two positive solutions.

For an arbitrary positive integer $n$, we can choose proper constants to meet expectation of $f$, so that problem (1.1) has at least $n$ positive solutions. 
Theorem 4.3 Assume that there are constants $0<c_{1}<c_{2}<c_{3}<\cdots<c_{2 n-1}<c_{2 n}$ such that

(i) $\max _{0 \leq v \leq c_{i}} f(t, v+\varphi(t)) \leq \frac{c_{i}}{\lambda I_{2}}$ for all $t \in[0,1], i=1,4, \ldots, 4 k-3+\frac{3\left(1+(-1)^{n}\right)}{2}$ and

(ii) $\min _{\frac{1}{4} c_{i} \leq v \leq c_{i}} f(t, v+\varphi(t)) \geq \frac{c_{i}}{\lambda I_{1}}$ for all $t \in[0,1], i=2,3, \ldots, 4 k-2+\frac{1+(-1)^{n}}{2}$,

where $k=1,2, \ldots,\left[\frac{n+1}{2}\right]$. Then the boundary value problem (1.1) has at least $n$ positive solutions.

\section{Competing interests}

The authors declare that the study was realized in collaboration with the same responsibility. All authors read and approved the final manuscript.

\section{Authors' contributions}

The authors declare that they have no competing interests.

\section{Acknowledgements}

The authors sincerely thank the reviewers for their valuable suggestions and useful comments that have led to the present improved version of the original manuscript. This research is supported by the Natural Science Foundation of China (11571202, 61374074), Natural Science Outstanding Youth Foundation of Shandong Province (JQ201119) and supported by Shandong Provincial Natural Science Foundation (ZR2012AM009, ZR2013AL003).

Received: 8 January 2015 Accepted: 23 October 2015 Published online: 16 November 2015

\section{References}

1. Nagy, Á: Density functional. Theory and application to atoms and molecules. Phys. Rep. 298, 1-79 (1998)

2. Ourabah, K, Tribeche, M: The nonextensive Thomas-Fermi theory in an n-dimensional space. Physica A 392 4477-4480 (2013)

3. Kılıçman, A, Hashimb, I, Kajani, MT, Maleki, M: On the rational second kind Chebyshev pseudospectral method for the solution of the Thomas-Fermi equation over an infinite interval. J. Comput. Appl. Math. 257, 79-85 (2014)

4. Kilbas, AA, Srivastava, HH, Trujillo, JJ: Theory and Applications of Fractional Differential Equations. Elsevier, Amsterdam (2006)

5. Oldham, KB, Spanier, J: The Fractional Calculus. Academic Press, New York (1974)

6. Podlubny, I: Fractional Differential Equations. Academic Press, New York (1999)

7. Guo, D, Lakshmikantham, V: Nonlinear Problems in Abstract Cones. Academic Press, Orlando (1988)

8. Krishnan, B, Jayakumar, K: On the controllability of fractional dynamical systems. Int. J. Appl. Math. Comput. Sci. 22, 523-531 (2012)

9. Kaczorek, T: Selected Problems of Fractional Systems Theory. Lecture Notes in Control and Information Sciences, vol. 411. Springer, Berlin (2011)

10. Machado, JAT: Analysis and design of fractional-order digital control systems. Syst. Anal. Model. Simul. 27, 107-122 (1997)

11. Mozyrska, D, Pawluszewicz, E: Controllability of $h$-difference linear control systems with two fractional orders. In: 13 th International Carpathian Control Conference (ICCC), pp. 501-506 (2012)

12. Mozyrska, D, Pawluszewicz, E: Local controllability of nonlinear discrete-time fractional order systems. Bull. Pol. Acad. Sci., Tech. Sci. 61(1), 251-256 (2013)

13. Sierociuk, D, Nski, AD: Fractional Kalman filter algorithm for the states parameters and order of fractional system estimation. Int. J. Appl. Math. Comput. Sci. 16, 129-140 (2006)

14. Agarwal, RP, O'Regan, D: An upper and lower solution approach for a generalized Thomas-Fermi theory of neutral atoms. Math. Probl. Eng. 8(2), 135-142 (2002)

15. Zhang, S: Positive solutions for boundary-value problems of nonlinear fractional differential equations. Electron J. Differ. Equ. 2006, 36 (2006)

16. Sun, $\mathrm{H}$, Wen, W: On the number of positive solutions for a nonlinear third order boundary value problem. Int. J. Differ Equ. 1, 165-176 (2006)

17. Zhao, Y, Sun, S, Han, Z, Zhang, M: Positive solutions for boundary value problems of nonlinear fractional differential equations. Appl. Math. Comput. 217, 6950-6958 (2011)

18. Su, X, Zhang, S: Solutions to boundary-value problems for nonlinear differential equations of fractional order Electron. J. Differ. Equ. 2009, 26 (2009)

19. Zhai, C, Xu, L: Properties of positive solutions to a class of four-point boundary value problem of Caputo fractional differential equations with a parameter. Commun. Nonlinear Sci. Numer. Simul. 19, 2820-2827 (2014)

20. Sun, S, Li, Q, Li, Y: Existence and uniqueness of solutions for a coupled system of multi-term nonlinear fractional differential equations. Comput. Math. Appl. 64, 3310-3320 (2012)

21. Feng, W, Sun, S, Han, Z, Zhao, Y: Existence of solutions for a singular system of nonlinear fractional differential equations. Comput. Math. Appl. 62, 1370-1378 (2011)

22. Sun, S, Zhao, Y, Han, Z, Li, Y: The existence of solutions for boundary value problem of fractional hybrid differential equations. Commun. Nonlinear Sci. Numer. Simul. 17, 4961-4967 (2012)

23. Zhang, Q, Jiang, D: Upper and lower solutions method and a second order three-point singular boundary value problem. Comput. Math. Appl. 56, 1059-1070 (2008)

24. Zhao, Y, Sun, S, Han, Z, Li, Q: The existence of multiple positive solutions for boundary value problems of nonlinear fractional differential equations. Commun. Nonlinear Sci. Numer. Simul. 16, 2086-2097 (2011)

25. Feng, W, Sun, S, Li, X, Xu, M: Positive solutions to fractional boundary value problems with nonlinear boundary conditions. Bound. Value Probl. 2014, 225 (2014) 